\title{
Implicit Reconstruction of Vasculatures Using Bivariate Piecewise Algebraic Splines
}

\author{
Qingqi Hong*, Qingde Li, and Jie Tian, Fellow, IEEE
}

\begin{abstract}
Vasculature geometry reconstruction from volumetric medical data is a crucial task in the development of computer guided minimally invasive vascular surgery systems. In this paper, a technique for reconstructing the geometry of vasculatures using bivariate implicit splines is developed. With the proposed technique, an implicit geometry representation of the vascular tree can be accurately constructed based on the voxels extracted directly from the surface of a certain vascular structure in a given volumetric medical dataset. Experimental results show that the geometric representation built using our method can faithfully represent the morphology and topology of vascular structures. In addition, both the qualitative and the quantitative validations have been performed to show that the reconstructed vessel geometry is of high accuracy and smoothness. An virtual angioscopy system has been implemented to indicate one of the strengths of our proposed method.
\end{abstract}

Index Terms-Implicit modeling, vasculature reconstruction, virtual angioscopy.

\section{INTRODUCTION}

A CCURATE reconstruction of vessel geometry is an important task in the field of medical data visualization [1]. It plays a crucial role in the area of computer-aided diagnosis and computer guided minimally invasive vascular surgery, such as the diagnosis of anomalous growths and stenosis [2], virtual angioscopy [3]. Though the vessel structures contained in a volume dataset can be visualized using certain direct volume rendering techniques and the image generated in this way can be quite useful and suitable for the task of computer-aided vascular diagnosis, in developing a computer-aided vascular surgery system, just to be able to visualize the hidden vascular structures is far from sufficient. We are required not only to see the objects but also to touch and feel them. Obviously, it can be very difficult to accurately locate and position the vascular objects directly from the image generated from direct volume rendering. This is because without an actual geometry representation for the vascular structures, it can be an

Manuscript received July 03, 2011; revised September 25, 2011; accepted October 04, 2011. Date of publication October 18, 2011; date of current version March 02, 2012. This workwas supported by the National Basic Research Program of China (973 Program) under Grant 2011CB707700. Asterisk indicates corresponding author.

*Q. Hong is with the Department of Computer Science, University of Hull, HU6 7RX Hull, U.K. (e-mail: hongqq@gmail.com).

Q. Li is with the Department of Computer Science, University of Hull, HU6 7RX Hull, U.K. (e-mail: q.li@hull.ac.uk).

J. Tian is with the Medical Image Processing Group, Institute of Automation Chinese Academy of Sciences, Beijing 100190, China (e-mail: tian@ieee.org)

Color versions of one or more of the figures in this paper are available online at http://ieeexplore.org.

Digital Object Identifier 10.1109/TMI.2011.2172455 extremely challenging task to register such an image with the actual vascular objects, which is a fundamental task required in the process of computer-aided vascular surgery. In this paper, we choose to reconstruct the vascular geometry as an implicit surface, since the isosurfaces extracted directly from the segmented vasculature using surface rendering techniques (i.e., Marching Cubes [4]) are usually poor, frequently exhibiting artifacts and discontinuities. In this research, we aim to construct the vessel geometry accurately and smoothly as an implicit function from a cloud of voxels extracted from the segmented vessel surface. The most basic requirement of a vasculature reconstruction technique is that the reconstructed geometry must be accurate. The requirement of accuracy for reconstructing 3-D vascular tree is obviously imperative for any computer systems involving diagnosis and computer-aided surgery [5]. In addition, the reconstructed surfaces are expected to be of high-level smoothness and can be expressed in an analytical form. An explicit analytical expression of vasculature not only allows to display the reconstructed surface with any specified resolution, but can also be very useful in the stage of vessel analysis, such as the measurement of blood flux, vascular shape analysis, and the orientation for the specific portions of the vessel structure. All these tasks could be quite difficult to be achieved based on the segmentation result since it is a discrete point set [6].

Due to the complex nature of vascular structures, the best way to represent the topology of vasculature is to use skeleton curves. The skeleton of an object, which is identified as the locus of the centers of maximal spheres inside the object [7], has the ability to naturally capture important shape characteristics in three-dimensional contexts [8]. Thus, the skeleton-based reconstruction is regarded as the most natural option to construct efficiently the complete vascular structures [9]. Various skeleton-based methods have been proposed for reconstructing vasculatures from segmented dataset. However, most of these approaches are model-based, assuming that a vascular lumen is having a certain regular shape, such as cylinders [10], truncated cones [11]. Although these methods can achieve certain level of smoothness in a relatively fast speed, they are far from accurate as the model used in the reconstruction process is usually too ideal to correctly represent the actual variation presented by the cross sections of a vascular structure. As a result, the reconstructed geometry cannot be used in a computer-aided surgery system by surgeons for performing computer-aided minimally invasive vascular surgery. In addition, the vascular surfaces generated from most of these methods are represented either in parametric form or as polygonal mesh, which are prone to generate artifacts and discontinuities at branching where graphics 
primitives are fitted together [1]. Oeltze and Preim [6] have applied the convolution surfaces [12] for the reconstruction of vasculatures, which can achieve quite smooth vessel surfaces even at branchings. However, this method is based on an ideal model assumption that the cross sections of vascular structures are all circular. Actually, the cross sections of vessels are not always circular, especially for that of pathologic vessels.

In this paper, we present a novel approach for the accurate reconstruction of vasculature along its skeleton without model assumptions. This technique is based on an implicit surface modeling method that we have developed to model generalized cylinders [13]. In this implicit generalized cylinders modeling method, the freeform cross sections are first reconstructed implicitly using the 2-D piecewise algebraic splines [14], and then, different cross-section profiles are weighted and summed up along the skeleton using the partial shape preserving (PSP) spline basis functions [15], the 1-D version of 2-D piecewise algebraic splines. In addition, we employ the smooth piecewise polynomial blending operations [16] to blend the branches of implicitly constructed generalized cylinders together. Our method can construct a smooth, $C^{m-1}(m \geq 2)$ continuous implicit surface represented explicitly in analytic form, which can be evaluated extremely efficient [14]. The proposed method has been applied to actual 3-D medical data for the reconstruction of vasculatures. The experiment results show that the reconstructed vascular shapes are of high accuracy and smoothness. Some qualitative and quantitative analysis has been carried out to show the validation of proposed technique. A virtual angioscopy system is implemented using the reconstructed implicit vascular surface to demonstrate one of the strengths of our method.

\section{RELATED WORK}

Surface reconstruction approaches for vasculatures can be grouped into two categories: skeleton-based surface reconstruction and skeleton-free surface reconstruction. The methods in the latter category directly reconstruct vascular surfaces from segmented data without the usage of pre-extracted skeleton of the vessel. Marching Cubes (MC) [4] is the most commonly used surface construction technique of this kind. However, the quality of the constructed images of vasculature based on MC is relatively low due to the use of linear interpolation for filling the gap between neighboring voxels [5]. Although simple smoothing procedures can be employed to reduce the aliasing artifacts of surface visualization, the appropriate smoothing of vascular structures is a challenging task and does not lead to the desired results in general [17]. Another issue associated with MC is that it is error-prone and does not always guarantee a correct reconstruction of the vasculature morphologically and topologically. Constrained elastic surface nets (CESN) [18] is the best general method to smooth visualizations of binary segmentation results, but it still induces unsatisfactory result for small vascular structures [5].

Schumann et al. [5], [19] have proposed a method based on multi-level partition of unity (MPU) implicits [20], which provides a superior quality of visualizing results when compared to CESN. The basic principle of this method is to convert the segmentation result into a point cloud that is transformed into a surface representation by means of MPU Implicits, which fits a smooth quadratic surface locally to each local subdataset. Generally, the implicit surfaces are able to represent a given geometry smoothly without explicitly constructing the geometry [1]. However, due to the complex and fine nature of most vascular tree, the quality of the resulting surfaces is usually poor and has to be improved using an additional remeshing step [19], either based on parameterization, or fitting of subdivision surfaces [21], which subsequently increases the effort required from the reconstruction process and inevitably introduces further errors.

As discussed in the first section, it would be more intuitive and appropriate to reconstruct the surface along its skeleton for tubular structure. Various skeleton-based methods have been proposed for reconstructing vasculatures from segmented dataset. When perform skeleton-based vascular surface reconstruction, the first thing needed to be done is to determine the vessel centerline and the local vessel cross sections [1]. The generation of skeleton is usually achieved by the technique of morphological thinning, or a step-by-step approach, which moves a small sub-volume, such as parallelepiped or sphere, to recursively slide along the vessel tree [22]. Once the skeleton has been extracted out from the input segmented data, the explicit geometry models of vasculatures can be constructed by using the geometric information provided for the vessel cross sections. When only diameter is available for the vessel cross section, certain model-based approaches can be used to construct the vascular surfaces by using certain type of geometric primitives, such as cylinders [10], truncated cones [11]. However, the quality of the vascular surfaces generated using this method depends on the number of vertices used to approximate the cross-section profile and the number of geometric primitives associated with the skeleton [1]. Although these methods can achieve certain level of smoothness in a relatively fast speed, when the model is incorrect, which is often the case, they may lead to geometric surfaces that are quite unreal. In addition, most of the geometric surfaces reconstructed in these ways are represented as a kind of polygonal mesh, which are prone to producing artifacts and discontinuities at branching when geometric primitives are blended together.

Various shape modeling techniques have been suggested to construct geometric shapes along skeletons such that the constructed shape can achieve smooth transition at the points of branching. Hohne et al. have proposed to use B-spline surfaces to approximate small vascular structures and nerves [23]. Felkel et al. have proposed a method based on the subdivision of an initial coarse base mesh [24]. Bornik et al. have employed simplex meshes for the high-quality visualization of vascular structures [25]. However, all methods above are explicit model based. They either express the underlying geometric shape as a polygonal mesh or as a parametric surface. As have been pointed out above, they are difficult to perform shape blending operations in general. Recently, Wu et al. [9] have improved the Felkel et al. meshing method to achieve relatively accurate and smooth vascular structures. However, this approach is based on the fitting of explicit circles or ellipses to the cross sections of vessel, which in general cannot provide an accurate approximation to the actual vascular objects. In addition, the underlying shape is 
expressed as polygonal mesh, which has a difficulty of implementing ramification of branchings [26].

Another way to represent the underlying geometric shape is to use an implicit function, such as the methods proposed in Bloomenthal's work [12], [27]. An implicit surface is a surface that consists of those points $\boldsymbol{P}$ satisfying the implicit function, $f(\boldsymbol{P})=0$ [28]. One way to construct an implicit surface along a given skeleton is to use the convolution surfaces (CS) [12], which has been employed in [6] to visualize vasculatures. This method, taking input of vessel skeleton and the diameter information per voxel, produces smooth transitions at branchings and closed, rounded ends. The technique of CS visualization can usually show much better visual quality, when compared with the truncated cone visualization. However, the reconstructed vascular geometry is only a morphological approximation, which is far from accurate and does not meet the basic requirements of computer-aided vascular diagnosis and computer guided vascular surgery, due to its simplifying model assumption of circular cross sections.

\section{IMPLICIT GENERALIZED CYLINDERS BASED ON 2-D PieCEWISE Algebraic Splines}

The skeleton-based reconstruction of vasculatures is to construct a geometric surface by sweeping one or more cross sections along the skeleton [29]. This kind of surfaces has been frequently referred to as generalized cylinders. Generally, the generalized cylinders can be either represented explicitly as parametric surfaces or polygonal meshes, or implicitly as implicit functions [26]. The explicit representation usually suffers from the problem of cross-sections intersection when the skeleton is too curved, and has a difficulty of implementing the ramification of one cylinder into two or more [26]. Implicit generalized cylinders represented by distance surface [30] or convolution surfaces [12] are limited to the assumption of circular shape for cross sections. The implicit swept surfaces introduced in [29], [31] using profile curves are limited to "star shape" due to the polar definition [32]. Though some more flexible techniques were proposed to generate an implicit sweeping surface [32]-[34] without the shape limitation of cross sections, the optimization for the swept shapes remains a difficult problem due to the respecting various constrains [35]. In this paper, we propose a technique to model implicit generalized cylinders based on 2-D piecewise algebraic splines [14], which allows one to reconstruct generalized cylinders with arbitrary cross sections. Our method is based on smooth blending of a set of locally constructed general cylinders corresponding to different cross sections along a given skeleton. The implicit generalized cylinders constructed using our method can achieve any required accuracy and continuity. Moreover, the implicit surface generated in this way can have an explicit analytic representation. The detailed descriptions of our proposed method are as follows.

\section{A. The Calculation of the Frenet Frame for the Skeleton}

Frenet frame is a locally specified reference frame, defined along a curve using the curve's tangent $\boldsymbol{T}$, normal $\boldsymbol{N}$, and binormal $\boldsymbol{B}$. Suppose $\boldsymbol{S}(s)$ is a parametric curve representing the
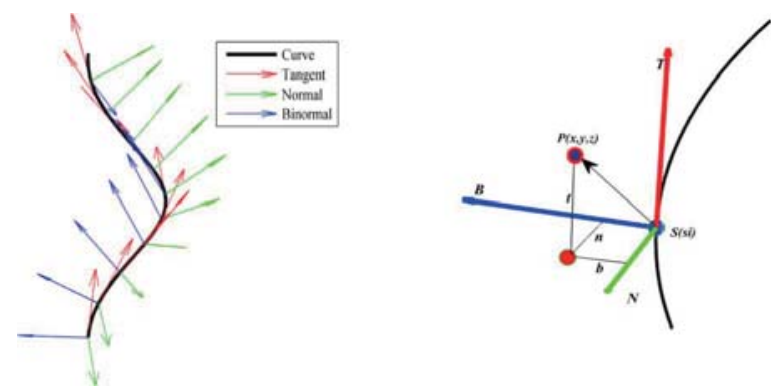

Fig. 1. Frenet frames of the sampling points on a parametric curve (left), and the transformation of coordination to the local Frenet frame (right).

skeleton of a geometric shape, when $\boldsymbol{S}(s)^{\prime}$ is not 0 , the definitions of $\boldsymbol{T}, \boldsymbol{N}$, and $\boldsymbol{B}$ are given by

$$
\left\{\begin{array}{l}
\boldsymbol{T}(s)=\boldsymbol{S}(s)^{\prime} /\left\|\boldsymbol{S}(s)^{\prime}\right\| \\
\boldsymbol{N}(s)=\boldsymbol{S}(s)^{\prime \prime} /\left\|\boldsymbol{S}(s)^{\prime \prime}\right\| . \\
\boldsymbol{B}(s)=\boldsymbol{N}(s) \times \boldsymbol{T}(s)
\end{array} .\right.
$$

For each point on the skeleton, its Frenet frame can be computed conveniently. As shown in Fig. 1 (left), the black curve represents the skeleton, and the red arrow, green arrow, as well as blue arrow, respectively, represents the tangent vector, normal vector, and binormal vector of the point on the skeleton. For a curve, there might be some "bad" points at which the curvatures of the curve are zeros. In this case, we utilize the $\boldsymbol{T}, \boldsymbol{N}$, and $\boldsymbol{B}$ vectors of their adjacent points instead. If the curvature is always zero then the curve will be a straight line. And it is easy to define the orthonormal basis of $\boldsymbol{R}^{3}$ for a straight line.

\section{B. The Transformation of Coordinates to the Frenet-Frame Space}

Suppose $\boldsymbol{P}(x, y, z)$ is a point in 3-D space $\boldsymbol{R}^{3}$, and then for a skeletal point $\boldsymbol{S}\left(s_{i}\right)$, the coordinates of $\boldsymbol{P}$ can be transformed to the orthonormal basis of $\boldsymbol{R}^{3}$ defined by the local Frenet frame at $\boldsymbol{S}\left(s_{i}\right)$. As shown in Fig. 1 (right), $\boldsymbol{P}(x, y, z)$ is transformed to $\boldsymbol{P}_{f}(b, n, t)$ by the following equations:

$$
\left\{\begin{array}{l}
b(\boldsymbol{P})=\left(\boldsymbol{P}-\boldsymbol{S}\left(s_{i}\right)\right) \cdot \boldsymbol{B}\left(s_{i}\right) \\
n(\boldsymbol{P})=\left(\boldsymbol{P}-\boldsymbol{S}\left(s_{i}\right)\right) \cdot \boldsymbol{N}\left(s_{i}\right) \\
t(\boldsymbol{P})=\left(\boldsymbol{P}-\boldsymbol{S}\left(s_{i}\right)\right) \cdot \boldsymbol{T}\left(s_{i}\right)
\end{array}\right.
$$

where $\boldsymbol{B}\left(s_{i}\right), \boldsymbol{N}\left(s_{i}\right)$, and $\boldsymbol{T}\left(s_{i}\right)$ are unit vectors representing the binormal, normal, and tangent of the Frenet frame at $\boldsymbol{S}\left(s_{i}\right)$.

\section{Implicit Specification of Freeform Cross Section Using the 2-D Piecewise Algebraic Splines}

2-D piecewise algebraic spline was introduced by Li and Tian in [14] for the purpose of freeform implicit shape modeling. It is a generalization of the conventional parametric spline technique, whose basis functions have all the good properties of the conventional B-spline basis functions, such as non-negativity, partition of unity and convex-hull property [14].

For a given control polygon specified in the $b n$-coordinate plane of the Frenet frame, an implicit curve can be constructed by $B_{\triangle, \delta}^{(m)}(b, n)=h$, where $B_{\triangle, \delta}^{(m)}(x, y)$ is the 2-D piecewise algebraic spline. $(b, n)$ represents the $b n$-coordiantes of a point in 3-D space with respect of the Frenet frame. $\triangle$ is the control 

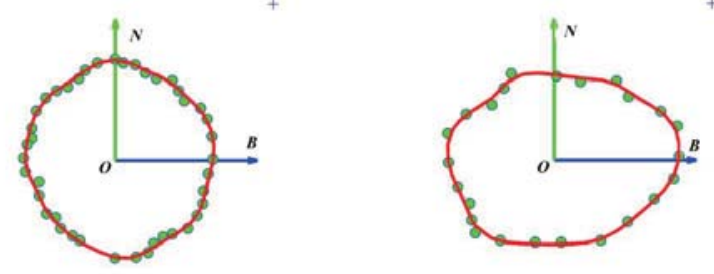

Fig. 2. Freeform implicit curves designed by 2-D piecewise algebraic spline: $B_{\triangle, \delta}^{(m)}(b, n)=h$.

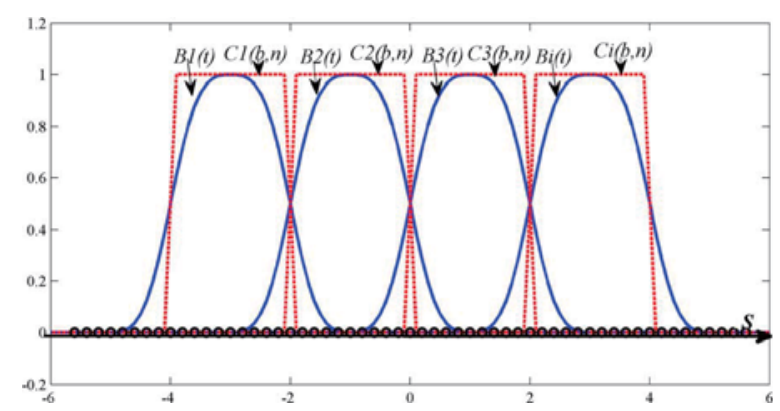

Fig. 3. The construction of implicit function $f(b, n, t)$ by weighted summing a set of implicit cross sections $B_{i}(b, n)$ along a skeleton with PSP-spline basis function $B_{i}(t)$.

polygon. $\delta$ is the polygon smooth parameter. $m$ is the degree of smoothness of the required bivariate function. $h$ is the height level. Fig. 2 demonstrates the implicit curves designed in this way.

The 2-D implicit curve that defines the cross section of the required shape at $\boldsymbol{S}\left(s_{i}\right)$, can be considered as a 3 -D implicit surface by mapping 3-D Space $\boldsymbol{R}^{3}$ to the local Frenet frame: $b=X(x, y, z), n=Y(x, y, z)$ according to (2). Suppose the cross-section profile is defined as an implicit function $C(b, n)=$ $B_{\triangle \delta}^{(m)}(b, n)-h=0$ in the $b n$-coordinate plane of the Frenet frame, then the extruded implicit surface $F(x, y, z)$ can be given by

$$
\begin{aligned}
F(x, y, z) & =C(X(x, y, z), Y(x, y, z)) \\
& =B_{\triangle, \delta}^{(m)}(X(x, y, z), Y(x, y, z))-h=0 .
\end{aligned}
$$

\section{Construction of Freeform Implicit Surface Along Skeleton With Variable Cross Sections}

As shown in [14], freeform implicit surfaces can be designed as a weighted sum of a set of 2-D implicit control curves along a coordinate axis (i.e., z-axis) by PSP-spline basis functions. With the introduction of Frenet frame, different cross-section profiles can be weighted and summed up together along an arbitrary skeleton, not limited to coordinate axis, to form an implicit generalized cylinder with variable cross sections. The required generalized cylinder can be described implicitly in the Frenet-frame space by the following form (see Fig. 3):

$$
f(b, n, t)=\sum_{i=1}^{L} C_{i}(b, n) B_{i}(t)=0
$$
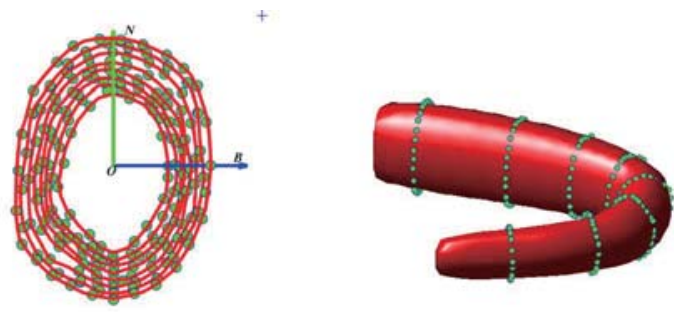

Fig. 4. The construction of implicit generalized cylinder (right) by weighted summing eight cross sections (left) along a skeleton.

where $b, n$, and $t$ are defined, respectively, in the Frenet-frame space according to $(2) ; i=1,2, \ldots, L$, in which $L$ is the amount of cross sections. Suppose $s_{i}$ is a list of knots for the parametric position $s$ of skeleton $S(s)$, then $C_{i}(b, n)$ is the implicitly defined cross section corresponding to the skeletal point $\boldsymbol{S}\left(s_{i}\right)$, and $B_{i}(t)$ is the spline basis function defined at $\boldsymbol{S}\left(s_{i}\right)$ along the tangent vector. Suppose $d_{i}$ is the distance between $\boldsymbol{S}\left(s_{i}\right)$ and $S\left(s_{i+1}\right)$, then $B_{i}(t)$ in (4) can be expressed in the following form:

$$
B_{i}(t)=B_{\left[-d_{i} / 2, d_{i} / 2\right], \delta}^{(m)}(t)
$$

where the general PSP-spline basis function $B_{[a, b], \delta}^{(m)}(x)$ is defined in the following way [14]:

$$
B_{[a, b], \delta}^{(m)}(x)=H_{m}\left(\frac{b-x}{\delta}\right)-H_{m}\left(\frac{a-x}{\delta}\right)
$$

where $[a, b]$ is an interval with $a \leq b$; and $H_{m}(x)$ is the smooth unit step function introduced in [36].

From (2), it can be seen that $b, n$, and $t$ in (4) relating to the Frenet frame at each $\boldsymbol{S}\left(s_{i}\right)$ are all functions of $x, y, z$. That is, $b=X(x, y, z), n=Y(x, y, z)$, and $t=Z(x, y, z)$. Therefore, the generalized cylinder constructed according to (4) can be expressed as follows:

$$
F(x, y, z)=f(X(x, y, z), Y(x, y, z), Z(x, y, z))=0 .
$$

As presented in Fig. 4, the implicit generalized cylinder is constructed as a weighted sum of eight adjacent cross sections along a skeleton with PSP-spline basis functions.

\section{E. Blending for the Branches of Implicit Shapes}

The technique presented above is only appropriate when no branches exist for the given skeleton. In the case of branching, an implicit general cylinder is first constructed from each individual branch, and then all these branching implicit general cylinders can be blended together to represent the overall implicit surfaces corresponding to the entire structure. There are various ways to blend a set of implicit surfaces, but most of them are achieved with nonpiecewise-polynomial shape operators and without the flexibility in controlling the blending range. Since the implicit surfaces from different branches of the skeleton are piecewise polynomial, piecewise polynomial shape blending operations are expected. In this paper, we use the extended smooth maximum function introduced in [16] to blend implicit shapes constructed from different skeletal 

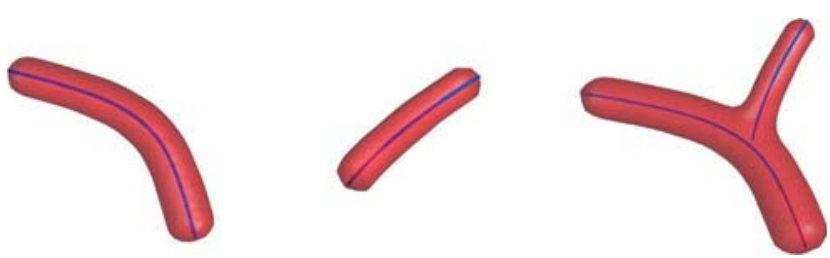

Fig. 5. Smooth blending of two implicitly defined shapes with $\max _{n, \delta}(x, y)$. Two individual implicit objects (left and middle) are smoothly blended as one implicit object (right) by using the smooth maximum function $\max _{n, \delta}(x, y)$.

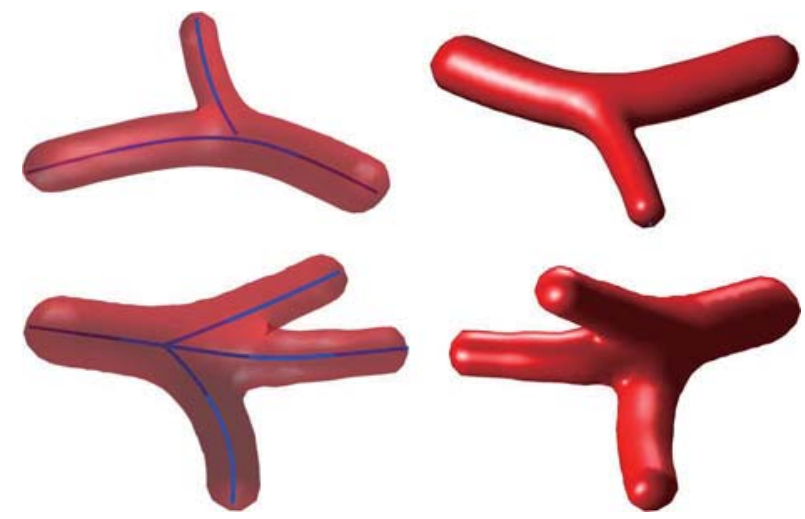

Fig. 6. Smooth and bulge-free blending of implicit generalized cylinders using piecewise polynomial blending operations.

branches (see Fig. 5). The extended smooth maximum function is defined in the following form:

$$
\max _{n, \delta}(x, y)=\frac{1}{2}\left(x+y+|x-y|_{n, \delta}\right)
$$

where $|x|_{n, \delta}$ is the smooth absolute function with the smoothness degree $n$ and blending range-control parameter $\delta$ defined in [16]. This kind of implicit shape blending operation is piecewise polynomial and allows flexible blending range specification.

Many implicit blending operations have a problem of over-blending, which may exhibit bulges around the point of branching [6]. Our approach inherits the weighted summation mechanism to generate smooth surface with different cross sections for the same skeletal element. In the case of branching, we can easily achieve bulge-free blending by utilizing smooth blending operation $\max _{n, \delta}()$ to blend the branches of implicit surfaces for different skeletal elements. As is shown in Fig. 6, our method can achieve smooth and bulge-free blending at branchings.

\section{ACCURATE RECONSTRUCtion OF VASCULATURES}

In this section, we apply the method proposed above for the purpose of vascular structure reconstruction, which basically involves the extraction of the contour points along each branch of the constructed skeleton and the implicit reconstruction of vascular structures using the extracted contours.

\section{A. The Extraction of Control Points for the Specification of Accurate Cross Sections}

After the process of segmentation [37], the shape of vessel can be identified as the voxels with intensive value $\geq 0$. Before constructing the implicit generalized cylinder for the vascula-
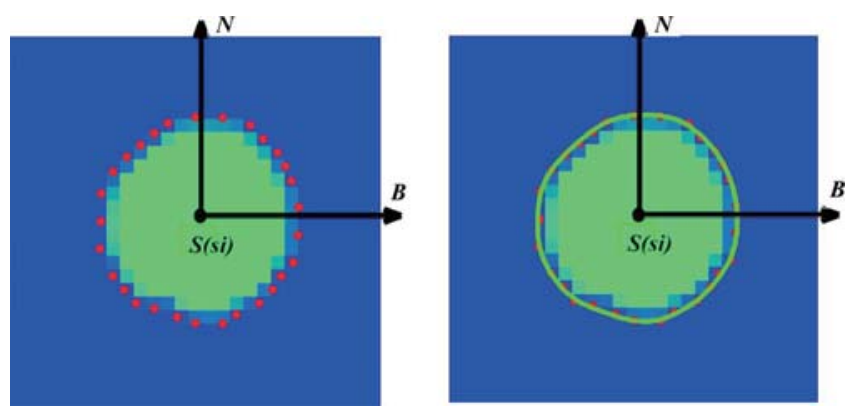

Fig. 7. The extraction of contour points with zero intensive value (left), and the smooth implicit curve specified by the extracted contour points (right).

tures, we need to extract along the skeleton a set of points for specifying the accurate cross sections of the vascular vessel to be constructed. Suppose $\boldsymbol{S}\left(s_{i}\right)$ is a skeletal point, then the steps for extracting the control points for specifying the cross section corresponding to $\boldsymbol{S}\left(s_{i}\right)$ are as follows.

1) Define a rectangle based on the local coordinate system.

2) Transform the coordinate of the rectangle to its world coordinate system, and intersect with the vessel surface.

3) Map the intensive value of the segmented vessel into the defined rectangle.

4) Extract the contour points with zero intensive value. As shown in Fig. 7 (left), the red points represent the extracted contour points with zero intensive value.

Generally, the side length of the rectangle is set as three times of the cross-section radius. If the cross section of the vessel excesses the area of the defined rectangle, the rectangle is considered to be intersecting with more than one vessel branches. In this case, we drop some of the target points outside the inscribed circle of the rectangle to insure that the target area is closed. Most of the dropped target points are in the area of the cross section of the unintended branch. Although the target area may still includes a certain part of the unintended branch, this does not affect the final reconstruction result, since the area will be blended with the vessel structure constructed from other surrounding branch(es).

\section{B. The Accurate Reconstruction of Vascular Structures}

Once the contour points for specifying the cross sections of vessel have been extracted, we can employ our proposed implicit generalized cylinder to model the vasculatures accurately. The modeling steps are as follows.

1) Suppose $S_{j}(s)$ is a skeletal branch of the vessel tree. For each skeletal point $\boldsymbol{S}_{j}\left(s_{i}\right)$, the coordinates of the arbitrary point in 3-D space $\boldsymbol{R}^{3}$ are transformed into the space defined by the local Frenet frame at $\boldsymbol{S}_{j}\left(s_{i}\right)$ (please refer to Section III-B).

2) For each skeletal point $\boldsymbol{S}_{j}\left(s_{i}\right)$, we employ the method proposed in Section IV-A to extract the control points for specifying the cross section around $\boldsymbol{S}_{j}\left(s_{i}\right)$.

3) Based on the extracted control points, a smooth curve corresponding to cross section $C_{i}$ can then be implicitly constructed using 2-D piecewise algebraic splines (please refer to Section III-C) [see Fig. 7 (right)]. 

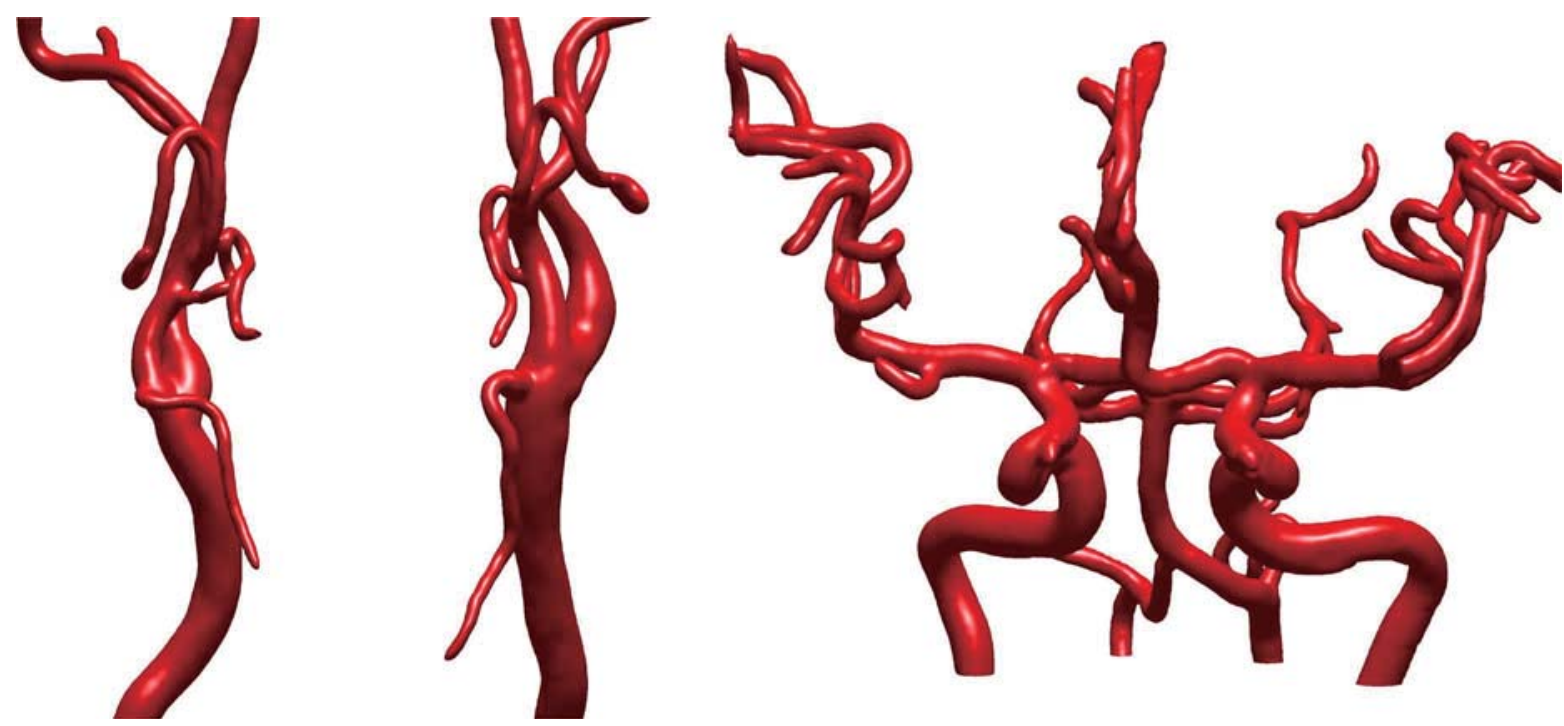

Fig. 8. Some of the reconstruction results using our method: CTA carotid artery (left); MRA cerebral vasculatures (right).

4) Using the idea presented in Section III-D, different cross-section profiles specified at each skeletal points are then weighted and summed up together along the skeleton $\boldsymbol{S}_{j}(s)$, to form an implicit generalized cylinder representing the vessel branch. $d_{i}$ in (5), that is the distance between the skeletal point $\boldsymbol{S}\left(s_{i}\right)$ and $\boldsymbol{S}\left(s_{i+1}\right)$, is defined to be proportional to the radius and in inverse proportion to the curvature of the current cross-section profile. In the case of much curved skeleton, $d_{i}$ should be set quite small to guarantee the smooth blending of different cross-section profiles. As an alternative, we can extrude a 2-D specified cross-section profile into 3-D implicit surfaces along the implicitly fitted curve to the skeleton [14].

5) Finally, the implicitly defined vessel surfaces built according to different skeletal branches $\boldsymbol{S}_{j}(s)(j=$ $1,2, \ldots, N)$ are blended together using the smooth maximum function $\max _{n, \delta}(x, y)$ to construct the complete vascular tree (please refer to Section III-E).

\section{Results And Discussions}

\section{A. Reconstruction Results Based on Our Method}

The medical datasets used in our first three experiments are provided by Intelligent Bioinformatics Systems Division, Institute of Automation, the Chinese Academy of Sciences, in the format of DICOM (Digital Imaging and Communications in Medicine). The first example is the reconstruction of carotid artery (CA) for the 3-D CT angiography (CTA) images with a resolution of $512 \times 512 \times 206$ and spacing of $0.52 \mathrm{~mm} \times 0.52$ $\mathrm{mm} \times 0.63 \mathrm{~mm}$. The second example is the reconstruction of cerebral vasculatures $(\mathrm{CV})$ for the 3 -D magnetic resonance angiography (MRA) images with a resolution of $352 \times 448 \times 114$ and spacing of $0.49 \mathrm{~mm} \times 0.49 \mathrm{~mm} \times 0.80 \mathrm{~mm}$. The third example is the reconstruction of abdominal aorta (AA) for the 3-D MRA images with a resolution of $512 \times 512 \times 300$ and spacing of $0.70 \mathrm{~mm} \times 0.70 \mathrm{~mm} \times 0.63 \mathrm{~mm}$. The last example is the reconstruction of the segmented liver portal vein (LPV) using a medical dataset obtained from the public resource 1 which has a resolution of $512 \times 512 \times 310$ and spacing of $0.78 \mathrm{~mm} \times 0.78$ $\mathrm{mm} \times 1.60 \mathrm{~mm}$. As can be seen from Fig. 8, a visual inspection shows that our proposed method can correctly represent the morphology and topology of vascular structures. In addition, very thin branches and curved, complex structures can be reconstructed faithfully using our method.

For comparisons, Fig. 9 shows a detailed look at the reconstructed cerebral vessels. As is presented in the figure, the isosurface rendering of the binary data (top left) suffers from strong aliasing artifacts like staircases, which has a strong divergence with real vessels and might hamper the visual interpretation of the vessel surface [19]. Although the segmentation result based on level set method [37] (top right) can achieve certain smooth surface when compared to the binary data, the visualization result is still poor, and needs further smoothing steps. The reconstruction method based on MPUI (bottom left) can construct smooth surface with certain visual quality. However, due to the complex and fine nature of most vascular tree, the quality of the resulting surfaces is still not good enough and has to be improved using an additional remeshing step [19], which subsequently increases the effort required from the reconstruction process and inevitably introduces further errors. In contrast, our approach can achieve superior visual quality and produce smooth transitions at branchings (bottom right). In fact, by using the proposed method, the vascular surfaces can be built to have any required geometric continuity. Furthermore, much more accurate vessel surfaces can be built since our method does not involve model assumptions. Generally, our method can achieve highly smooth and accurate vessel surfaces, which are quite faithful to the actual vessels.

\section{B. Validation}

The validation of any reconstruction technique is very crucial for its clinical applications [6]. In this section, we investigate the strengths of the proposed technique from both the

${ }^{1}$ http://www.ircad.fr/softwares/3Dircadb/3Dircadb1/index.php 

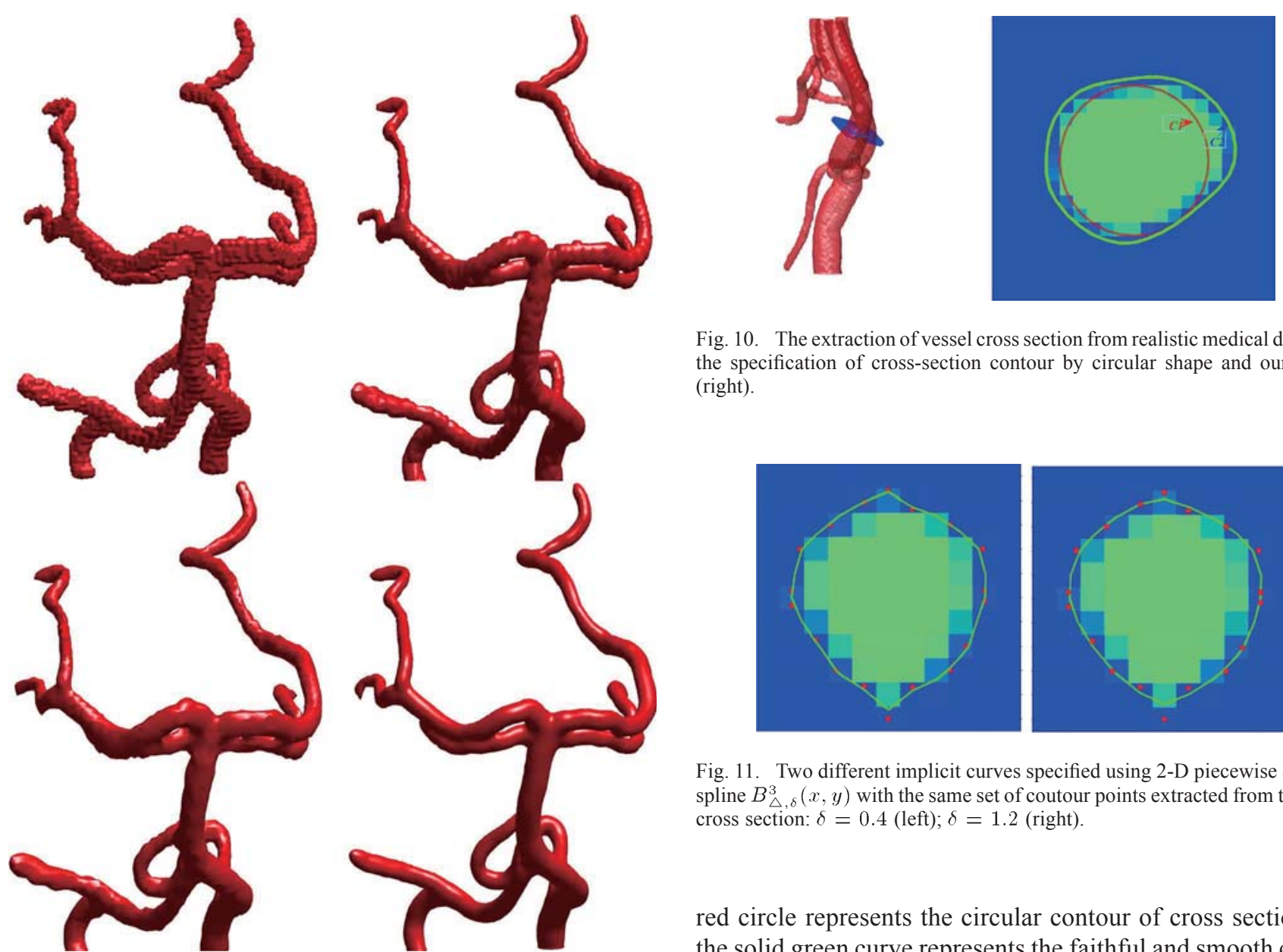

Fig. 10. The extraction of vessel cross section from realistic medical data (left); the specification of cross-section contour by circular shape and our method (right).
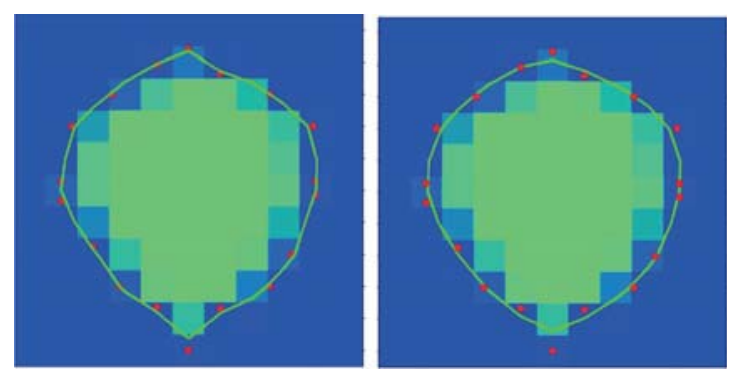

Fig. 11. Two different implicit curves specified using 2-D piecewise algebraic spline $B_{\triangle, \delta}^{3}(x, y)$ with the same set of coutour points extracted from the vessel cross section: $\delta=0.4$ (left); $\delta=1.2$ (right).

Fig. 9. A detail look at the reconstruction of the MRA cerebral vessels: the isosurface rendering of binary data (top left), the segmentation result using level set method (top right), the reconstruction result using MPU Implicits (bottom left), and our method (bottom right).

qualitative and quantitative points of view using the segmented vessel datasets. Our goal is to reconstruct the continuous vessel surfaces from the segmented point sets. A good reconstruction method is considered to approximate the "original" vessel as accurately as possible. Furthermore, the smoothness of the reconstructed surface is also a basic requirement since it is the essential feature of the actual vessel surfaces.

1) Qualitative Validation: Generally, our proposed approach has two key ingredients to guarantee the requirements of accuracy and smoothness. First and foremost, the cross sections of the vessel are freely specified by 2-D implicit splines, without any model assumption, such as circular or elliptical shapes. The freeform specification of the vessel cross sections is an essential requirement for computer-aided vessel diagnosis, since the cross sections of vascular geometry are not always circular, especially for those pathologic vessels. As can be seen from Fig. 10, the technique based on our method can represent the cross section of vessel more faithfully. In fact, the implicit curve constructed using 2-D implicit splines can achieve any preset precision, as long as the smoothing parameter is sufficiently small. In addition, by choosing the parameter of continuous degree $m$, the implicit curve can achieve any required continuity $C^{m-1}(m \geq 2)$. In Fig. 10 (right), the dashed red circle represents the circular contour of cross section, and the solid green curve represents the faithful and smooth contour of cross section specified by our method.

However, this does not mean that the smoothing parameter $\delta$ should be set as small as possible. Because for a certain resolution, if $\delta$ is set too small, the constructed curve as well as the surface would not be as smooth as the realistic vessel surface in visual inspection, especially in the case of reconstructing from noisy dataset. Fig. 11 demonstrates the two different implicit curves specified using 2-D piecewise algebraic spline $B_{\triangle, \delta}^{3}(x, y)$ with the same set of contour points extracted from the vessel cross section. Implicit curve presented on the left is constructed with $\delta=0.4$, which is much closer to the "original" point sets, but with less smoothness. Although the smoothness can be improved by increasing the spatial resolution, it would cost much more time to display the cruve/surface. On the other hand, the implicit curve presented on the right is constructed with $\delta=1.2$, which is not so close to the "original" point sets as to the left curve, but it is much smoother than that of left curve displaying on the same resolution to represent the realistic vessel cross section. Therefore, to some extent, a trade off needs to be made between the faithfulness of the reconstructed surface to the "original" point sets and the smoothness of its appearance. In this paper, the $\delta$ is generally set as 0.8 , which can guarantee a reasonable trade-off between accuracy and smoothness.

The other key ingredient is that we employ a partial shape preserving (PSP) spline basis functions [14], [15] to smoothly combine the collection of implicit control surfaces. As stated in [15], the PSP-spline basis function not only has all the advantages of 


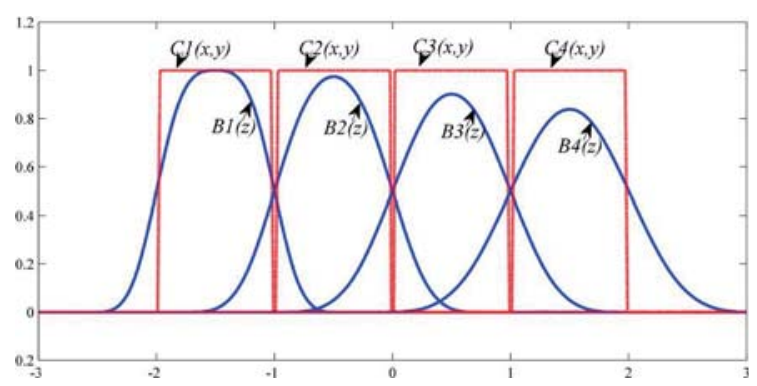

Fig. 12. The blending of a set of implicit cross sections $C_{i}(x, y)$ with different PSP-spline basis functions $B_{i}(z)$. From left to right, the controlling parameter $\delta$ in $B_{i}(z)$ is changed from 0.5 to 1.1 with an increasing interval 0.2 .
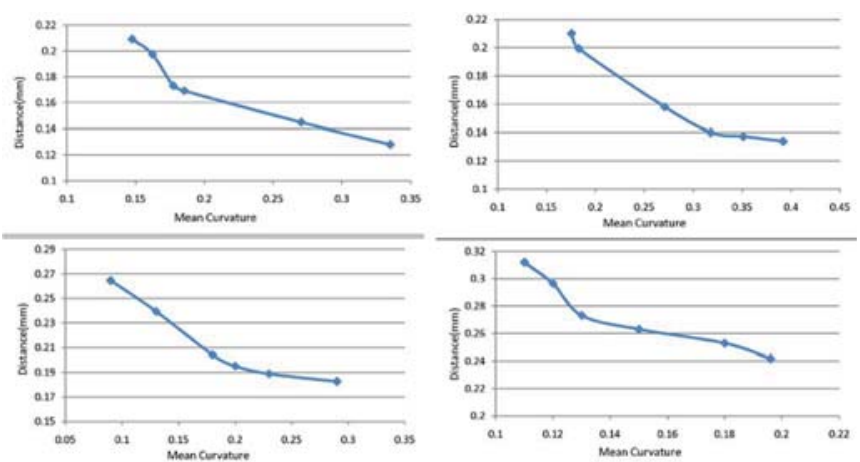

Fig. 13. Quantitative analysis for our reconstructed surfaces. The correlation between the measured distances and mean curvatures for: the carotid artery constructed from CTA dataset (top left), the cerebral vessels constructed from MRA dataset (top right), the abdominal aorta constructed from MRA dataset (bottom left), the liver portal vein from binary segmentation result (bottom right).

the conventional B-spline technique, but it is also a shape-preserving spline, which can preserve the original cross-section surfaces as much as possible. In addition, it is very flexible to specify the blending range of shapes by simply adjusting the smoothing parameter. Fig. 12 shows the blending of a set of cross sections $C_{i}(x, y)$ with different PSP-spline basis functions $B_{i}(z)$.

2) Quantitative Validation: Besides the visual inspection, the quantitative validation analysis is necessary for estimating whether the underlying data (segmented vessel dataset) are faithfully represented with certain smoothness [6]. For judging the accuracy of reconstruction, we analyse the distances between segmented data and our reconstructed surface. The comparison is realized in the following way: for each vertex on the isosurface of the segmentation result, the Euclidean distance to its closest point on our reconstructed surface is calculated. For estimating the smoothness, we examined the mean curvature of each vertex on the reconstructed surface, since the mean curvature has the ability of getting insight to the degree of smoothness of the surface [38].

Curve in Fig. 13 illustrates the correlation between the measured distances and mean curvatures for our experimental datasets. The vertical axis represents the mean distances of each vertex on the isosurface of the segmentation result to our reconstructed surface; and the horizontal axis represents the average of the unsigned mean curvatures (AUMC) for all voxels. As demonstrated in the curve, the minimum deviation can be as small as $0.12 \mathrm{~mm}$ with certain smoothness (the AUMC is
TABLE I

QUANTITATIVE COMPARISON BETWEEN OUR RECONSTRUCTED SURFACES AND THAT BASED ON MPUI METHOD. DISTANCES ARE GIVEN IN MILLIMETERS. THE ACCURACY IS MEASURED BY CALCULATING THE MEAN DISTANCES BETWEEN THE SEGMENTATION RESULT TO THE RECONSTRUCTED SURFACE, AND THE SMOOTHNESS IS ESTIMATED BY MEASURING THE AUMC OF THE RECONSTRUCTED SURFACE

\begin{tabular}{ccccc}
\hline \hline Datasets & \multicolumn{2}{c}{ MPUI based method } & \multicolumn{2}{c}{ Our method } \\
& Dis(mm) & AUMC & Dis(mm) & AUMC \\
\hline CA & 0.27 & 0.29 & 0.17 & 0.21 \\
CV & 0.27 & 0.35 & 0.16 & 0.28 \\
AA & 0.44 & 0.27 & 0.21 & 0.19 \\
LPV & 0.37 & 0.20 & 0.27 & 0.15 \\
\hline
\end{tabular}

less than 0.4) and the maximum deviation cannot be bigger than $0.31 \mathrm{~mm}$ even the level of smoothness is set quite high. As discussed in the last section, the accuracy and the smoothness of the reconstructed surface from the discrete point sets needs to be balanced. Generally, our method can achieve highly accurate reconstructed surface, of which the mean deviation is as small as $0.20 \mathrm{~mm}$, which is quite smaller than the mean distance $(0.39 \mathrm{~mm})$ presented in [6] and the median of the deviations $(0.30 \mathrm{~mm})$ presented in [19]. Furthermore, the mean deviation is much less than half of the mean diagonal voxel size $(1.28 \mathrm{~mm})$ of the used datasets $(0.96 \mathrm{~mm}$ for the CTA carotid artery dataset, $1.05 \mathrm{~mm}$ for the MRA cerebral vessel dataset, $1.17 \mathrm{~mm}$ for the MRA abdominal aorta dataset, and $1.94 \mathrm{~mm}$ for the segmented liver portal vein). In addition, the surfaces are reconstructed with quite satisfied smoothness, not only in visual inspection but also the quantitative analysis (the AUMC is generally as small as 0.207 ).

The quantitative comparison has also been conducted between the surfaces reconstructed using our method and that based on MPUI method. As have been shown in Table I, the mean deviations and AUMC corresponding to the surfaces built from our method are much smaller than that for the surfaces reconstructed based on the MPUI method for each experimental data set. In other words, our method can achieve more accurate and smoother vessel structures than the MPUI based method.

\section{Computational Complexity}

Generally, a skeletal branch is long. Thus, it is necessary to partition the space along the skeleton for the purpose of accelerating the computation. In other words, the long skeleton is divided into several sub-skeletons, and for each sub-skeleton, an axis-aligned bounding box is computed in voxel coordinates (see Fig. 14). Then for each sub-skeleton, its local implicit function is constructed within its corresponding axis-aligned bounding box. Finally, all of the local implicit functions are summarized together to form the global implicit function representing the vessel structure reconstructed from the whole skeletal branch. In addition, we employ the NVIDIA CUDA (compute unified device architecture) [39] to evaluate each local implicit function value in GPU, which can greatly reduce the visualization time.

To demonstrate the complexity of our algorithm, Table II presents the information concerning the complexity of the resulting models and the reconstruction and visualization time of our implicit surfaces based on the medical datasets used above, 

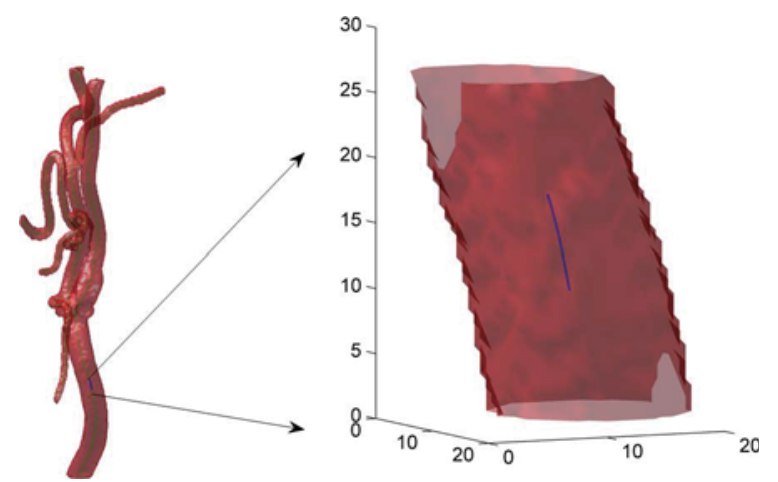

Fig. 14. Subskeleton subdivided from the whole skeleton (left), and its corresponding axis-aligned bounding box (right).

TABLE II

PERFORMANCE MEASUREMENTS FOR THE IMPLICIT SURFACE RECONSTRUCTION FROM ANATOMIC VASCULAR STRUCTURES CARRIED OUT ON AN AMD ATHLON CPU 4600+, 2.41 GHz SYSTEM WITH 2.00 GB RAM AND NVIDA GEFORCE 8600 GT GRAPHICS CARD WITH 32 STREAM PROCESSORS AND $540 \mathrm{MHZ}$ FREQUENCY

\begin{tabular}{ccc}
\hline \hline Datasets & Number of faces & Time (second) \\
\hline CA & 133116 & 86.37 \\
CV & 157018 & 143.99 \\
AA & 155224 & 165.30 \\
LPV & 112980 & 189.52 \\
\hline
\end{tabular}

which includes the time of point extraction, construction of implicit functions, evaluation of scalar values, and generation of patch faces.

\section{Reconstruction for Pathological Vasculatures}

Our technique has also been applied for the reconstruction of pathological vasculatures from the patient data sets with vessel disease. The first example is the reconstruction of carotid artery with stenosis for the 3-D CTA images with a resolution of $512 \times 512 \times 100$ and spacing of $0.48 \mathrm{~mm} \times 0.48 \mathrm{~mm} \times 0.65$ $\mathrm{mm}$ [see Fig. 15 (left)]. As can be seen from the rectangle area, our algorithm can faithfully represent the stenosis of carotid artery and achieve superior visual quality.

Another example is the reconstruction of peripheral artery with aneurysm for the 3-D CTA images with a resolution of $512 \times 512 \times 240$ and spacing of $0.83 \mathrm{~mm} \times 0.83 \mathrm{~mm} \times 1.00$ $\mathrm{mm}$ [see Fig. 15 (right)]. As shown in the rectangle area, our technique can accurately reconstruct the peripheral artery with aneurysm in the form of implicit functions, which would be helpful to perform the quantitative analysis for the pathological vessel.

\section{E. Virtual Angioscopy}

The accurate reconstruction of vascular tree is very crucial for virtual angioscopy, a noninvasive medical diagnosis procedure for exploring the human vascular system [40], which generates an interactive environment for the vascular examination from a point of view inside the vessels [41]. Actually, virtual fly-through of vascular structures is a useful technique for educational purposes and some diagnostic tasks, as well as intervention planning and intraoperative navigation [1]. In general,
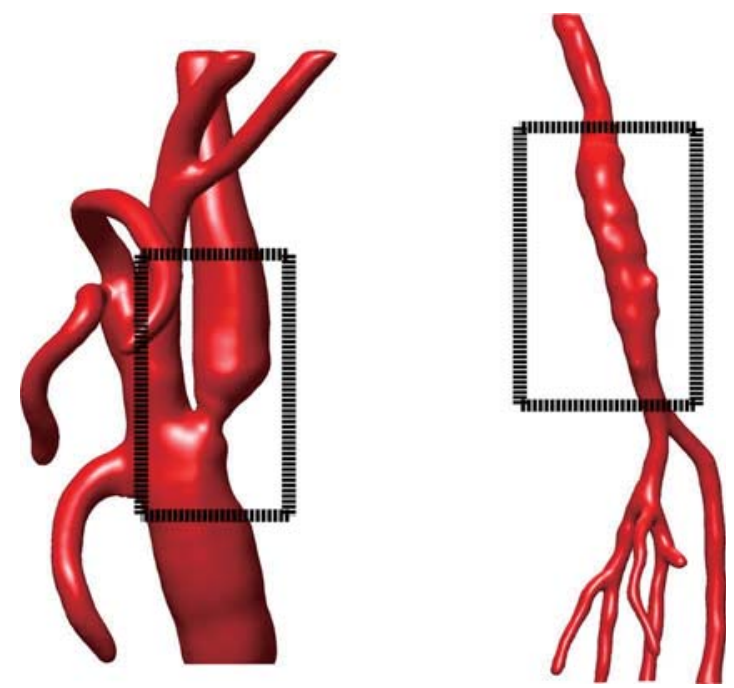

Fig. 15. The reconstruction of pathological vasculatures: carotid artery with stenosis(left), and peripheral artery with aneurysm (right).

it is essential to combine detailed views of the inner structures with an overview of the anatomic structures (see Fig. 16).

One of the main issues associated with the virtual angioscopy is the requirement of high visual quality of perspective view inside the dataset. Generally, the common approach for the visualization of a virtual angioscopy is surface rendering, yielding images close to a real endoscopy. However, the direct application of surface rendering algorithms (i.e., Marching Cubes) to the segmented vasculatures may suffer from the typical diamond shaped artifacts [see Fig. 16 (right)] caused by the trilinear interpolation [42]. Therefore, the smooth and accurate reconstruction of vascular tree is very crucial for virtual angioscopy. Based on our implicit representation of vasculatures, the surface rendering methods can achieve high quality perspective views as well as accurate cross sections (without model assumption) [see Fig. 16 (middle)], which is suitable for training purposes and diagnosis tasks.

Besides rendering, the camera navigation paradigm is another key problem required to be solved for the development of a virtual angioscopy system. Various virtual angioscopy techniques can be roughly classified into three classes: automatic navigation, manual or free navigation and guided navigation [43]. The drawback of automatic navigation is the lack of interactivity, which means that user interaction is limited and the irrelevant regions cannot be easily skipped [42]. By manual navigation, user can completely control over all parameters of the virtual camera without any constraints. However, collision avoidance is required, which is costly query operation and easily to result in significant lags between interaction and rendering [42].

While based on the implicit vascular geometry, the collision avoidance for the camera navigation of virtual angioscopy can be easily solved, since our method can guarantee to define an implicit volume by replacing the equality in (7) with an inequality

$$
F(x, y, z)=f(X(x, y, z), Y(x, y, z), Z(x, y, z)) \geq 0 .
$$

That is, the vasculatures are represented as a global implicit function $F(x, y, z)$. When $F(x, y, z)=0$, it represents the 

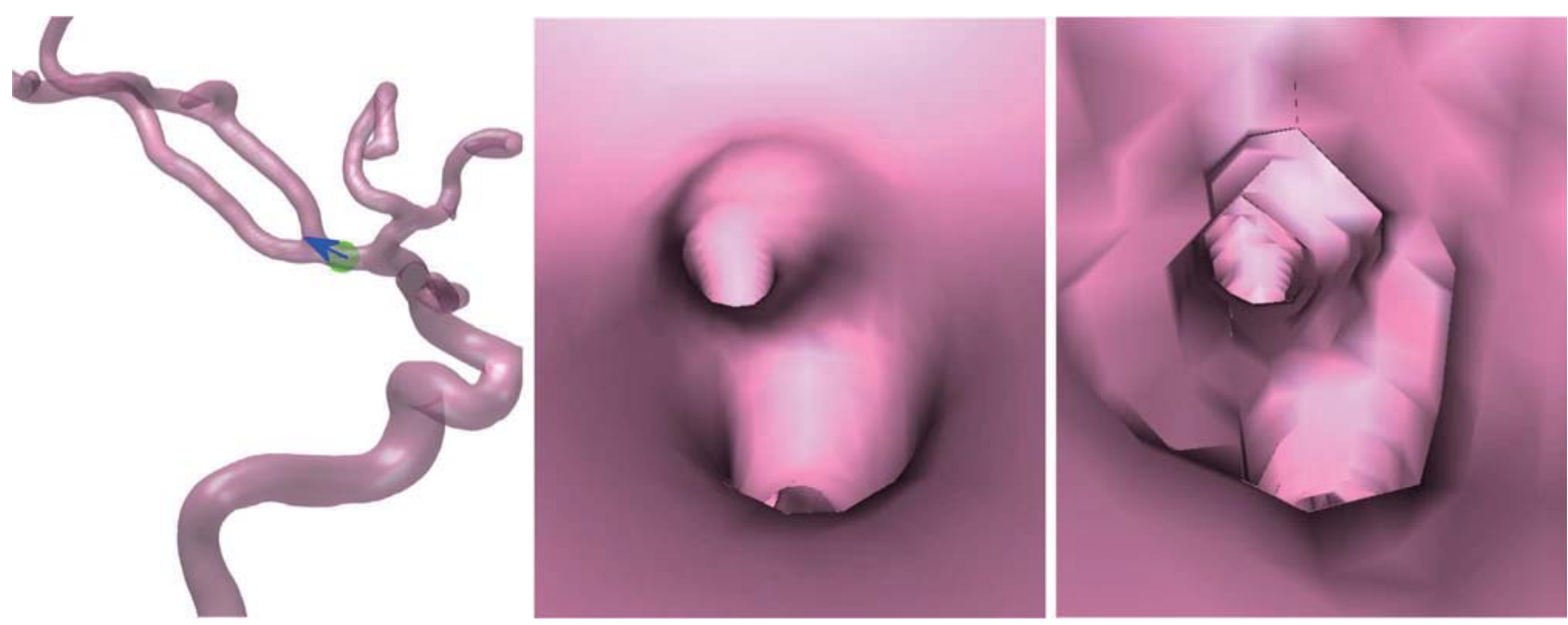

Fig. 16. Virtual angioscopy. The overview of the vessel structures, and the arrow indicating the current position and orientation of the camera (left); The perspective view inside the vessel based on our implicit modelling vasculatures (middle), and on direct application of Marching Cubes to the segmented vasculatures (right).

vessel surfaces; when $F(x, y, z)>0$, it represents implicit volume inside the vessel structures; and when $F(x, y, z)<0$, it represents the implicit volume outside the vessel structures. The implicit volume is a favourite kind of geometric object when performing collision detection [14]. When the vasculatures are modelled as implicit volume, one can tell directly whether a point lies inside or outside the vasculatures and the problem of collision detection can be easily solved [44].

\section{CONClusion AND Future Work}

In this paper, a technique for accurately reconstructing vascular structures has been presented, which is underpinned by a skeleton-based implicit generalized cylinder modeling method. With the proposed method, an implicit surface with extraordinary smoothness and accuracy can be constructed from a given segmented medical dataset. The experimental results and validations have shown that our method can achieve accurate, faithful and smooth vascular structures. In addition, virtual angioscopy has been implemented to demonstrate one of the strengths of our proposed method.

Our proposed method can accurately construct the continuous geometry of vasculatures in the form of implicit functions, which not only allows to achieve better visualization, but can also be very useful for the vessel shape analysis. Generally, our method has three main advantages over other techniques for reconstructing vasculatures. Firstly, the reconstructed surface is a kind of isosurface and can achieve extremely high smoothness and accuracy to the segmented discrete vascular surface points. Secondly, the modeling result of our method is an implicit volume, and can be directly applied for virtual angioscopy. Finally, the reconstructed implicit surfaces based on our method are easy to calculate, since there is an explicit analytic representation for the resulting shape.

It should be pointed out that the reconstruction accuracy of our method depends on the accuracy of segmentation results, since the implicit surfaces built with our method are based on the segmented data. So far, various segmentation methods have been developed, but the accurate segmentation of vasculatures still remains a challenging task, especially for noisy datasets. The development of highly accurate vascular-specific segmentation method will be one of our main tasks in the future. Another task that is worth of investigation in the future would be to apply the reconstructed vessel geometry for the simulation of computational fluid dynamics (CFD). A highly accurate and smooth vessel geometry representation is crucial for CFD to guarantee correct simulation results and to avoid numerical instabilities [19]. Simulations of the blood flow enable the study of hemodynamic characteristics such as intra-aneurysmal flow patterns or the wall shear stress, which plays an important role in the areas of predictive medicine and vascular disease study.

\section{ACKNOWLEDGMENT}

The authors would like to thank all the anonymous reviewers for their constructive comments.

\section{REFERENCES}

[1] B. Preim and S. Oeltze, "3-D visualization of vasculature: An overview," Visualizat. Med. Life Sci., pp. 39-59, 2007.

[2] A. Joshi, X. Qian, D. P. Dione, K. R. Bulsara, C. K. Breuer, A. J. Sinusas, and X. Papademetris, "Effective visualization of complex vascular structures using a non-parametric vessel detection method," IEEE Trans. Vis. Comput. Graphics, vol. 14, no. 6, pp. 1603-1610, Nov./Dec. 2008.

[3] D. Bartz, "Virtual endoscopy of the vascular system (virtual angioscopy)," [Online]. Available: http://www.gris.uni-tuebingen.de/people/staff/bartz/proj/endos/angioscopy.html 2003

[4] W. E. Lorensen and H. E. Cline, "Marching cubes: A high resolution 3-D surface construction algorithm," in Proc. ACM SIGGRAPH, 1987, pp. $163-169$.

[5] C. Schumann, S. Oeltze, R. Bade, B. Preim, and H.-O. Peitgen, "Model-free surface visualization of vascular trees," in IEEE/Eurograph. Symp. Vis., 2007, pp. 283-290.

[6] S. Oeltze and B. Preim, "Visualization of vascular structures with convolution surfaces: Method, validation and evaluation," IEEE Trans. Med. Imag., vol. 25, no. 4, pp. 540-548, Apr. 2005.

[7] C. Arcelli and G. Sanniti di Baja, "Euclidean skeleton via centre-of-maximal-disc extraction," Image Vis. Comput., vol. 11, no. 3, pp. 163-173, 1993.

[8] O. Faugeras, Three-Dimensional Computer Vision: A Geometric Viewpoint. Cambridge, MA: MIT Press, 1993. 
[9] X. Wu, V. Luboz, K. Krissian, S. Cotin, and S. Dawson, "Segmentation and reconstruction of vascular structures for 3-D real-time simulation,' Med. Image Anal., pp. 22-34, 2010.

[10] Y. Masutani, K. Masamune, and T. Dohi, "Region-growing-based feature extraction algorithm for tree-like objects," in Proc. Visualizat. Biomed. Comput., 1996, vol. 1131, pp. 161-171.

[11] H. Hahn, B. Preim, D. Selle, and H. Peitgen, "Visualization and interaction techniques for the exploration of vascular structures," in IEEE Visualization, 2001, pp. 395-402.

[12] J. Bloomenthal and K. Shoemake, "Convolution surfaces," in Proc. ACM SIGGRAPH, 1991, vol. 25, pp. 251-256.

[13] G. Agin, Representation and description of curved objects Stanford Artif. Intell. Rep. Memo AIM-173, 1972.

[14] Q. Li and J. Tian, "2-D piecewise algebraic splines for implicit modeling," ACM Trans. Graphics, vol. 28, no. 2, 2009.

[15] Q. Li and J. Tian, "Partial shape-preserving splines," Comput. Aided Des., vol. 43, pp. 394-409, Apr. 2011.

[16] Q. Li, "Smooth piecewise polynomial blending operations for implicit shapes," Computer Graphics Forum, vol. 26, no. 2, pp. 157-171, 2007.

[17] R. Bade, J. Haase, and B. Preim, "Comparison of fundamental mesh smoothing algorithms for medical surface models," In Simulation and Visualisierung, pp. 289-304, 2006.

[18] F. Sarah and G. Frisken, "Constrained elastic surface nets: Generating smooth surfaces from binary segmented data," in Proc. of MICCAI. New York: Springer, 1998, vol. 1496, pp. 888-898.

[19] C. Schumann, M. Neugebauer, R. Bade, H.-O. Peitgen, and B. Preim, "Implicit vessel surface reconstruction for visualization and CFD simulation," Int. J. Comput. Assist. Radiol. Surg., vol. 2, no. 5, pp. 275-286, 2008.

[20] Y. Ohtake, A. Belyaev, M. Alexa, G. Turk, and H. Seidel, "Multilevel partition of unity implicits," ACM Trans. Graphics, vol. 22, pp. 463-470, 2003.

[21] T. Boubekeur, W. Heidrich, X. Granier, and C. Schlick, "Volume-surface trees," Comput. Graphics Forum, vol. 25, no. 3, pp. 399-406, 2006.

[22] C. Kirbas and F. Quek, "A review of vessel extraction techniques an algorithms," ACM Comput. Surv., vol. 36, no. 2, pp. 81-121, 2004.

[23] K. Hohne, B. Pflesser, and A. Pommert, "A realistic model of the inner organs from the visible human data," in Proc. of Medical Image Computing and Computer-Assisted Intervention. New York: Springer, vol. 1935, pp. 776-785.

[24] P. Felkel, I. Wegenkitt, and K. Buhler, "Surface models of tube trees," in Proc. Comput. Graphics Int., 2004, pp. 70-77.

[25] A. Bornik, B. Reitinger, and R. Beichel, "Reconstruction and representation of tubular structures using simplex meshes," in Proc. Winter School Computer Graphics (WSCG), 2005, pp. 61-65.
[26] J. Bloomenthal, "Skeletal design of natural forms," Ph.D. dissertation, Univ. Calgary, Calgary, Canada, 1995.

[27] J. Bloomenthal, "An implicit surface polygonizer," in In Graphics Gems IV. Boston, MA: Academic, 1994, pp. 324-349.

[28] J. Bloomenthal and B. Wyvill, "Interactive techniques for implicit modeling," Comput. Graphics, vol. 24, no. 2, pp. 109-116, 1990

[29] C. Grimm, "Implicit generalized cylinders using profile curves," In Implicit Surfaces 99, pp. 33-41, 1999.

[30] I. Faux and M. Pratt, Computational Geometry for Design and Manufacture. New York: Wiley, 1979.

[31] B. Crespin, C. Blanc, and C. Schlick, "Implicit sweep objects," Comput. Graphics Forum, vol. 15, no. 3, pp. 165-174, 1996.

[32] R. Schmidt and B. Wyvill, "Generalized sweep templates for implicit modeling," in GRAPHITE'05: Proc. 3rd Int. Conf. Comput.Graphics Interactive Techniques Australasia South East Asia, New York, 2005, pp. 187-196.

[33] L. Barthe, N. A. Dodgson, M. A. Sabin, B. Wyvill, and V. Gaildrat, "Two- imensional potential fields for advanced implicit modeling operators," Comput. Graphics Forum, vol. 22, no. 1, pp. 23-33, 2003.

[34] J.-K. Seong, G. Elber, and M.-S. Kim, "Trimming local and global self-intersections in offset curves/surfaces using distance maps," Computer-Aided Design, vol. 38, no. 3, pp. 183-193, 2006.

[35] S. Azernikov, "Sweeping solids on manifolds," in Proc. 2008 ACM Symp. Solid Physical Model., New York, 2008, pp. 249-255.

[36] Q. Li, J. G. Griffiths, and J. Ward, "Constructive implicit fitting," Comput. Aided Geom. Des., vol. 23, no. 1, pp. 17-44, 2006.

[37] S. Osher and N. Paragios, Geometric Level Set Methods in Imaging Vision and Graphics. New York: Springer Verlag, 2003.

[38] C. Dong and G. Wang, "Curvatures estimation on triangular mesh," J. Zhejiang Univ. Sci., vol. 6A, pp. 128-136, 2005.

[39] NVIDIA, "Cuda zone," [Online]. Available: http://www.nvidia.com/ object/whatiscudanew.html 2010

[40] E. Gobbetti, P. Pili, A. Zorcolo, and M. Tuveri, "Interactive virtual angioscopy," in Proc. 9th IEEE Visualization, 1998, pp. 435-438.

[41] D. Bartz, W. Straßr, M. Skalej, and D. Welte, "Interactive exploration of extra and intracranial blood vessels," in Proc. IEEE Visualization, 1999, pp. 389-392.

[42] D. Bartz, "Virtual endoscopy in research and clinical practice," Comput. Graphics Forum, vol. 24, no. 1, pp. 111-126, 2005.

[43] L. Hong, S. Muraki, A. Kaufman, D. Bartz, and T. He, "Virtual voyage: Interactive navigation in the human colon," in Proc. ACM SIGGRAPH, 1997, pp. 27-34.

[44] M. Lin and S. Gottschalk, "Collision detection between geometric models: A survey," in Proc. IMA Conf. Math. Surfaces, 1998, pp. 37-56. 